\title{
Transformar la educación superior y obligatoria a través de aprendizaje-servicio y mentoría*
}

\section{Transform Higher and Compulsory Education via Service-Learning and Mentoring}

\author{
Francisco D. Fernández-Martín ${ }^{a}$ \\ Universidad de Granada, España \\ ORCID: http://orcid.org/0000-0003-1272-1131 \\ José L. Arco-Tirado \\ Universidad de Granada, España \\ ORCID: http://orcid.org/0000-0002-8390-9724 \\ Mirian Hervás-Torres \\ Universidad de Granada, España \\ ORCID: http://orcid.org/0000-0002-8694-9393 \\ Luís C. Delgado-Pastor \\ Universidad de Granada, España \\ ORCID: http://orcid.org/0000-0002-7796-1999
}

a Autor de correspondencia. Correo electrónico:
fdfernan@ugr.es

Para citar este artículo: Fernández-Martín, F. D., ArcoTirado, J. L., Hervás-Torres, M., \& Delgado-Pastor, L. C. (2018). Transformar la Educación Superior y Obligatoria a través de Aprendizaje-Servicio y Mentoría. Universitas Psychologica, 17(4), 1-12. https://doi.org/10.11144/Javeriana.upsy17-4.teso

\section{RESUMEN}

El propósito de este trabajo fue determinar el impacto de un programa fundamentado en aprendizaje-servicio y mentoría sobre las competencias profesionales del alumnado universitario, el rendimiento escolar de los estudiantes y la satisfacción de los docentes-tutores de educación obligatoria. Para ello, 40 estudiantes universitarios, previamente entrenados y siempre supervisados por los responsables del programa, efectuaron 352 sesiones de mentoría con 20 escolares, 67 sesiones de coordinación con sus 13 docentes-tutores y 84 sesiones de asesoramiento familiar a sus respectivas familias. Los resultados muestran diferencias estadísticamente significativas en competencias del alumnado universitario, además de mejoras en rendimiento escolar y elevados niveles de satisfacción de los docentes-tutores. Esta intervención ha contribuido a mejorar la eficacia y eficiencia de la educación universitaria y obligatoria.

\section{Palabras clave}

aprendizaje-servicio; mentoría; enseñanza basada en competencias; rendimiento escolar.

\begin{abstract}
The purpose of this study was to measure the impact of a service-learning and mentoring program on university students professional competencies, school students performance and their teachers-tutors satisfaction. The intervention consisted of 352 mentoring sessions with compulsory education students, 67 sessions of coordination with their teachers-tutors and 84 sessions of family counseling, delivered by university students, who were previously trained and continuously monitored by the programs coordinators. The results yield significant statistical differences on the
\end{abstract}


university students' competencies, as well as a tendency to improve school performance by school students, and high levels of teachers-tutors' satisfaction. This intervention has contributed to improve the efficacy and efficiency of compulsory Education as well as the university training. Keywords

service-learning; mentoring; competencies based education; school performance.

A pesar de los avances que se han producido en España en los últimos años en materia de educación, los resultados procedentes de la evaluación del sistema de educación y formación (Instituto Nacional de Evaluación Educativa, 2016; Organisation for Economic Cooperation and Development [OECD], 2016) revelan una baja productividad del mismo, respecto al conjunto de países de la Unión Europea (UE). Estos resultados están influyendo directamente en la inserción laboral de los jóvenes, potenciando que un elevado número de ellos no pueda prosperar en su transición de la educación al empleo y de este a su integración social (Consejo Económico y Social de España [CESE], 2015; European University Association, 2015; OECD, 2016). Un ejemplo de las consecuencias socioeconómicas de esta productividad, se aprecia en los datos sobre desempleo entre los jóvenes con menor preparación y el coste que implicará para España, cercano al $1.4 \%$ del PIB (CESE, 2015). No obstante, las empresas consideran que el origen de no encontrar jóvenes cualificados no se puede limitar al abandono escolar temprano, es necesario considerar también la calidad de la formación que están recibiendo, pues no se adapta a la demanda de las empresas (Instituto de la Empresa Familiar, 2011). Por tanto, se puede manifestar que los jóvenes requieren un mayor nivel de cualificación, que no solo de formación, para reducir la tasa de desempleo (Van der Ende, Walsh, \& Ziminiene, 2014).

Estas parecen ser algunas de las razones por las que España ha seguido la estela de reformas impulsadas desde la UE para reformar y alinear los sistemas educativos, incentivado paralelamente la puesta en marcha de diversas medidas encaminadas a mejorar la igualdad de oportunidades, la calidad y la gestión educativa (Consejo Escolar del Estado, 2015). Ahora bien, entre las múltiples reformas adoptadas destacan los programas de trabajo estratégico "Educación y Formación 2010" y "Educación y Formación 2020", dirigidos a mejorar la calidad y eficacia del sistema educativo, a través de la adopción de un enfoque de enseñanza por competencias (Comisión Europea, 2011). Sin embargo, un análisis minucioso de la amplitud y profundidad de la implementación de tales reformas en España, arroja que estos cambios fundamentales, por diferentes razones (la incorporación tardía de la lista de competencias básicas del modelo europeo a la normativa educativa, los efectos de la crisis, etc.), aún no han llegado a la enseñanza y evaluación del sistema educativo (CESE, 2015).

Asimismo, con el propósito de progresar en la consecución de los objetivos del marco estratégico "Educación y Formación 2020" (Consejo de la Unión Europea, 2009), se plantea la necesidad de seguir implementando medidas dirigidas a aumentar la eficiencia de la inversión educativa, mejorar la calidad y la eficacia de la educación y la formación, fortalecer la profesión docente y la institución escolar, entre otras (Consejo Escolar del Estado, 2015). No obstante, la Comisión Europea (2012) otorga una especial importancia a dos de las principales carencias que presenta el sistema educativo y de formación español, y que están lastrando el desarrollo, crecimiento y competitividad del país: (a) la mejora de los resultados educativos de estudiantes con alto riesgo de abandono escolar temprano y bajo nivel de aptitudes básicas y (b) la revisión y consolidación del perfil profesional del docente.

En esta línea, los primeros niveles educativos parecen los más adecuados para mejorar los resultados escolares del alumnado con alto riesgo de abandono escolar temprano (European University Association, 2015). Por su parte, en el otro extremo, la universidad constituye otro área prioritaria, ante la necesidad de mejorar la formación que se ofrece al alumnado y favorecer la generación de nuevas ideas y conocimiento (OECD, 2016), sobre todo en las titulaciones relacionadas con la profesión docente. Es más, 
este cambio radical de escenario en la educación, con mayor atención a su calidad y eficiencia, está forzando el cambio de rol del profesorado universitario, empujándole a explorar métodos de instrucción con los que mejorar los resultados de aprendizaje del alumnado (Westover, 2012). Efectivamente, si los docentes tienden a adquirir las competencias profesionales más bien a través de la formación inicial, y la enseñanza es el elemento que más influye en los resultados de los estudiantes, además de mejorar su perfil de empleabilidad y reforzar la imagen de la universidad (CESE, 2015; Hayward, 2014), resulta evidente la necesidad de experiencias como la que se aborda en este trabajo, el Programa Comunidad-Universidad (PCU).

El PCU es una experiencia educativa innovadora internivelar que ofrece al alumnado de titulaciones universitarias relacionadas con la profesión docente la posibilidad de participar como mentores en actividades de aprendizaje de carácter co-curricular dirigidas a prevenir problemas de fracaso y/o abandono escolar temprano entre los estudiantes de educación obligatoria, por supuesto, en coordinación con sus docentes-tutores y familias, y bajo la supervisión en todo momento del profesorado universitario responsable del programa. Para ello, por el potencial teórico, práctico y aplicado que reúnen para hacer frente a los importantes retos educativos y formativos que plantea el sistema educativo español, como base del diseño de intervención del PCU se han incorporado dos metodologías educativas innovadoras y catalizadoras de la adquisición de competencias profesionalizantes (Able, Ghulamani, Mallous, \& Glazier, 2014; Hayward, 2014): (a) el aprendizaje-servicio, que integra los conocimientos y materias del currículo con el aprendizaje y la realización de trabajos útiles y solidarios en la comunidad, en colaboración con instituciones públicas y privadas (Tapia, 2006; Westover, 2012) y (b) la mentoría, en el que personas de diferente edad y/o con más conocimientos y/o habilidades, tras un proceso de formación y entrenamiento, a través de un marco de relación asimétrico exteriormente planificado por profesionales, facilitan ayuda y apoyo con un rol fijo a otras personas con menos conocimientos y/o habilidades con propósitos preventivos y/o de instrucción (Arco, Fernández, \& Fernández, 2011; DuBois \& Karcher, 2014).

Los programas que incluyen en su diseño de intervención estas metodologías poseen una larga tradición en el sistema educativo y de formación, principalmente por su alto nivel de eficacia, eficiencia y utilidad para resolver problemas de diversa índole (Arco et al., 2011). Entre los múltiples beneficios de esta forma de entender y practicar la educación, se han identificado, entre otros, mejoras del desempeño académico de los jóvenes que participan y de las habilidades sociales, aumento de indicadores de salud y competencias profesionales en el caso de estudiantes de todos los niveles educativos, así como acrecentar las habilidades profesionales y la satisfacción de los educadores implicados (Clayton, Bringle, \& Hatcher, 2013; Eby, Allen, Evans, Ng, \& DuBois, 2008; Westover, 2012). También existen evidencias sobre los beneficios que este tipo de programas tiene sobre el clima social de las instituciones que los adoptan y ponen en marcha, y también las comunidades se benefician al aumentar la estructura organizativa y la participación social de sus miembros (Billig, Root, \& Jesse, 2005; Bringle, Hatcher, \& Jones, 2011; Westover, 2012).

En este sentido, si con la adopción de este tipo de metodologías docentes y actividades co-curriculares se consigue reducir de manera significativa el fracaso escolar del alumnado de educación obligatoria y mejorar el perfil de empleabilidad del alumnado universitario, se podrá demostrar que algunos de los principales problemas del sistema educativo y de formación se pueden solucionar de manera eficiente y sostenible, contribuyendo a la consecución de los objetivos de "Educación y Formación 2020" (Consejo de la Unión Europea, 2009).

Por tanto, el propósito de esta investigación fue explorar los efectos del PCU para cualificar las competencias profesionales del alumnado de titulaciones universitarias relacionadas con la profesión docente, ofreciéndoles actividades de aprendizaje de carácter co-curricular con 
las que, paralelamente, se pretendió mejorar el rendimiento escolar en competencias curriculares entre el alumnado de educación obligatoria, siempre en coordinación con sus docentes-tutores e involucrando a sus familias de forma más activa en su educación. Las hipótesis que se establecieron fueron: (1) existirán diferencias estadísticamente significativas en las puntuaciones directas medias logradas en estrategias de aprendizaje y motivación por el alumnado universitario en la fase postest respecto a la fase pretest, como consecuencia de su participación en el PCU; (2) el alumnado universitario mejorará en términos estadísticamente significativos sus puntuaciones directas medias obtenidas en habilidades sociales en la fase postest respecto a la fase pretest, como resultado de su participación en el programa; (3) existen diferencias estadísticamente significativas en el rendimiento escolar del alumnado de educación obligatoria en las diferentes áreas curriculares en la fase postest respecto a la fase pretest después de su participación en el PCU y (4) como consecuencia de su participación en el programa, el $100 \%$ de los docentes-tutores estará dispuesto a participar en próximas ediciones del PCU.

\section{Método}

\section{Participantes}

La muestra estuvo compuesta por 73 participantes distribuidos en tres submuestras: 40 alumnos universitarios o mentores PCU, 20 alumnos de educación obligatoria o alumnos PCU y 13 docentes-tutores de dicho alumnado de educación obligatoria o docentes PCU. La submuestra de mentores PCU se constituyó con 26 alumnos del Grado de Maestro en Educación Primaria, 12 alumnos del Grado en Psicología y dos alumnos del Grado de Maestro en Educación Infantil de la Universidad de Granada (UGR). La media de edad que mostraban era de 21.28 años (rango de 19-28 años). Su distribución por sexo fue de 32 mujeres y 8 hombres. Por su parte, la submuestra de alumnos PCU se compuso de
19 alumnos de Educación Primaria y un alumno de Educación Secundaria Obligatoria de cuatro centros educativos de una localidad del área metropolitana de Granada (España). La media de edad que mostraban fue de 8.1 años (rango de 4 a 13 años). Por sexo, 6 fueron mujeres y 14, hombres. La submuestra de docentes PCU se formó con 13 docentes-tutores de educación obligatoria de los cuatro centros educativos participantes. La media de edad que mostraban fue de 49.32 años (rango de 38-64 años). Su distribución por sexo fue de 12 mujeres y un hombre.

El procedimiento de selección de la muestra se basó en un tipo de muestreo no probabilístico, de conveniencia (Martínez, 2010), con diferentes etapas de selección no aleatoria de unidades muestrales. En este sentido, la parte de la población diana a la que se tuvo acceso en la primera etapa fue la de la UGR y la de cuatro centros educativos de una localidad del área metropolitana de Granada. En la segunda etapa, se seleccionaron las titulaciones de la UGR y las etapas educativas de los cuatro centros de enseñanza en las que se implementó el PCU, y posteriormente se llevó a cabo el plan de divulgación y captación: (a) 2 sesiones grupales con el alumnado universitario de las titulaciones seleccionadas, en las que se les informó de las condiciones y beneficios del programa, a la vez que se solicitó su participación y (b) 18 sesiones grupales de información y coordinación con el resto de agentes educativos (responsables de la administración y centros locales y educativos) en las que se les informó sobre el programa, se confirmó su compromiso, se recogieron sus percepciones y propuestas y se solicitó su participación. En lo que respecta a la tercera etapa, 47 alumnos universitarios se inscribieron voluntariamente en el programa después de leer y aceptar las cláusulas establecidas en el acuerdo, completar el protocolo y pruebas estandarizadas para mentores PCU, mientras que 23 docentestutores inscribieron a 33 alumnos de educación obligatoria (un docente-tutor inscribió cuatro alumnos, dos a tres alumnos, tres a dos alumnos y 17 a un alumno), en diferentes momentos temporales a lo largo del curso escolar, después 
de leer y aceptar las cláusulas establecidas en la solicitud de participación para centros educativos, completar el protocolo para alumnos PCU, tramitar la solicitud de participación por parte de las familias y aportar una copia del boletín de calificaciones.

Finalmente, en la cuarta etapa, se seleccionaron las diferentes submuestras. Por un lado, a partir de los 47 alumnos universitarios inscritos, se seleccionaron los mentores PCU $(n=40)$ bajo los siguientes criterios: ostentar una nota media superior a dos puntos, presentar puntuaciones centiles iguales o superiores a 50 puntos en las diferentes escalas de las pruebas estandarizadas, demostrar interés, disponer de tiempo, asistir a la sesión del seminario de formación y superar los ejercicios prácticos de dicho seminario. Por otro lado, a partir de los 23 docentes-tutores y 33 alumnos de educación obligatoria inscritos, se seleccionaron los alumnos PCU $(n=20)$ y sus correspondientes docentes PCU $(n=13)$, considerando como criterios la fecha de inscripción en el programa y la disponibilidad de mentores PCU para su asignación. Los 10 docentes-tutores y 13 alumnos restantes se mantuvieron en lista de espera.

\section{Materiales}

\section{Acuerdo para mentores PCU}

Documento formado por 17 cláusulas y un apartado de conformidad, que fue elaborado a partir de modelos de otros estudios análogos (Arco et al., 2011). Su objetivo fue definir los derechos y deberes del alumnado universitario que se inscribiera en el programa.

\section{Protocolo para mentores PCU}

Autoinforme compuesto de 18 ítems de respuesta abierta y seis de diferentes alternativas, agrupados en tres áreas: información demográfica, académica y de interés. Se construyó considerando las instrucciones de otros trabajos similares (Arco et al., 2011;
The National Mentoring Partnership, 2015). Su objetivo fue obtener la información relevante del alumnado universitario inscrito en el programa.

\section{Solicitud de participación para centros educativos}

Documento formado por seis apartados: qué puedo esperar del PCU, confidencialidad, responsabilidades, límites, procedimiento de acceso y aceptación. Se elaboró tomando como referencia materiales empleados en otras investigaciones similares (Arco et al., 2011). Sus objetivos fueron definir los derechos y deberes de los centros educativos y docentes-tutores del alumnado que fuera derivado al programa y formalizar las autorizaciones pertinentes.

\section{Protocolo para alumnos PCU}

Autoinforme compuesto de 25 ítems de respuesta abierta y 13 de diferentes alternativas, agrupados en cuatro áreas: información institucional, demográfica y escolar del alumno y necesidades específicas de apoyo educativo del alumno. Se construyó a partir de modelos de otros estudios equivalentes (Arco et al., 2011; The National Mentoring Partnership, 2015). Su objetivo fue recoger la información demográfica, escolar y de interés del alumnado que fuera derivado al programa.

Solicitud de participación de las familias de alumnos PCU

Documento formado por seis apartados: qué puedo esperar del PCU, límites, confidencialidad, responsabilidades, procedimiento de acceso y aceptación. Se elaboró considerando ejemplos de otros trabajos (Arco et al., 2011). Sus objetivos fueron concretar los derechos y deberes de las familias del alumnado que fuera derivado al programa y disponer de las autorizaciones pertinentes. 
Motivated Strategies Learning Questionnaire ([MSLQ]; Pintrich, Smith, García, Ë Mckeachie, 1991)

Escala Likert constituida por 81 ítems de siete alternativas de respuesta agrupados en 15 escalas que conforman cinco componentes: estrategias cognitivas y metacognitivas, estrategias de regulación de recursos, expectativas y valor. Este instrumento se eligió por ser uno de los pocos que mide estrategias de aprendizaje y motivación en el alumnado universitario, además de presentar una fiabilidad (alfa entre 0.52 y 0.93 puntos) y validez bastante aceptables (justificación de estructura factorial y validez predictiva sobre rendimiento).

Escala de Habilidades Sociales ([EHS]; Gismero, 2000)

Instrumento formado por 33 ítems de cuatro alternativas de respuesta agrupados en seis subescalas: autoexpresión en situaciones sociales, defensa de los propios derechos como consumidor, expresión de enfado o disconformidad, decir no y cortar interacciones, hacer peticiones e iniciar interacciones positivas con el sexo opuesto. Esta escala se utilizó por ser una de las pruebas que comúnmente se emplea para evaluar las habilidades sociales en adolescentes y adultos debido a su alta fiabilidad (alfa 0.89 puntos en adultos) y aceptable validez (coeficiente de correlación entre puntuación global y autodescripciones de 0.53 puntos; coeficientes de correlación con adjetivos: entre -0.84 puntos agresividad y 0.84 puntos asertividad).

\section{Copia de expediente escolar}

Copia original del boletín de calificaciones del alumnado de educación obligatoria, que se usó para calcular y evaluar su rendimiento escolar.

\section{Cuestionario de docentes PCU}

Escala compuesta de siete ítems de estimación de 1 a 10, 11 de dos alternativas y cinco de respuesta abierta, agrupados en tres áreas: efectos del PCU sobre implicados, coordinación, satisfacción general y mejoras. Se elaboró tomando como referencia materiales de otros trabajos (Arco et al., 2011). Su objetivo fue recoger información sobre el funcionamiento y efectos del programa de PCU según los docentes-tutores.

\section{Cuaderno de trabajo para mentores PCU}

Material en el que se presentaba de forma estructurada las sesiones de mentoría y que fue creado por los responsables del programa a partir de otros materiales y trabajos de características similares (Arco et al., 2011; Bringle et al., 2011; Clayton et al., 2013; The National Mentoring Partnership, 2015). Se utilizó durante las sesiones de mentoría y el plan de seguimiento del programa.

\section{Diseño y procedimiento}

Los diseños metodológicos adoptados fueron de carácter cuasiexperimental, pretest-postest para las Hipótesis 1, 2 y 3 y postest para la Hipótesis 4 (Ato, 2010). En cuanto al procedimiento, una vez efectuado el análisis de necesidades, después de diseñar y planificar el programa, se desarrolló el muestreo anteriormente descrito. Seguidamente, en los términos que se establece en la literatura (Arco et al., 2011; Westover, 2012), se establecieron las parejas de mentores PCU (por afinidad entre ellos y disponibilidad horaria) y se llevó a cabo el emparejamiento de submuestras, es decir, la asignación de alumnos PCU y sus correspondientes docentes PCU y familias, a los mentores PCU. Posteriormente, se implementó el plan de intervención, de seguimiento y de evaluación de resultados, tal y como se describe a continuación, para finalmente efectuar la evaluación del mismo. 
El plan de intervención del PCU se compuso de los contenidos, procedimientos y actividades extraídos de la bibliografía especializada (Arco et al., 2011; Bringle et al., 2011; Clayton et al., 2013; The National Mentoring Partnership, 2015). En este sentido, la primera actividad del plan de intervención que se llevó a cabo fue el seminario de formación para mentores PCU. El objetivo principal de esta acción formativa fue potenciar en el alumnado universitario aquellos conocimientos declarativos, procedimentales, condicionales y actitudinales necesarios para desempeñar de la forma más eficaz y eficiente posible las funciones que el programa les atribuía. Para ello, en una sesión de cuatro horas de duración, se impartieron, a través de enseñanza expositiva y aprendizaje por descubrimiento guiado, los siguientes bloques de contenidos: (a) presentación del equipo responsable, participantes y plan de formación, (b) fundamentación científica, académica e institucional del PCU, (c) ejemplos de dificultades (de atención, concentración, comunicación) y problemas (de motivación, conducta, emocionales, familiares, inclusión social) en el alumnado derivado al PCU, (d) variables causales de las dificultades y problemas del alumnado derivado al PCU, (e) evaluación funcional, (f) profundización en el análisis funcional y necesidades específicas de apoyo educativo y ( $\mathrm{g}$ ) diseño y planificación de sesiones de mentoría.

La segunda actividad del plan de intervención, es decir, las sesiones de mentoría o actividades extraescolares de carácter co-curricular entre mentores PCU y alumnos PCU, se extendieron a lo largo del segundo y tercer trimestre del curso escolar con una frecuencia semanal y una duración de 90 minutos, aproximadamente, en los espacios habilitados especialmente para ello por el programa. Estas sesiones fueron principalmente de refuerzo escolar, dirigidas a identificar las posibles dificultades de aprendizaje en las diferentes áreas curriculares, mejorar la motivación, adaptar los materiales, aumentar la práctica y el repaso, etc. y de orientación personal, dirigidas a clarificar y establecer metas, planificar acciones y optimizar decisiones. Paralelamente, los mentores PCU implementaron las sesiones de mentoría con los docentes PCU y familias de los alumnos PCU, con una frecuencia quincenal, aunque determinada por su disponibilidad, y una duración aproximada de 30 minutos en los espacios habilitados. Las sesiones de información y coordinación con los docentes PCU se dirigieron a, por un lado, recoger la información necesaria para diseñar, planificar e implementar las sesiones de mentoría con los alumnos PCU, y así utilizar en ellas las estrategias, materiales y metodologías que los docentes empleaban en el aula, minimizando diferencias que pudieran interferir con el aprendizaje del alumnado. Y, por otro lado, mantener informados a los docentes PCU del comportamiento y progresos de los alumnos PCU y del programa. Por su parte, las sesiones de asesoramiento psicopedagógico con las familias, tuvieron como principal propósito dotar a los padres de los alumnos PCU de más información, conocimiento y habilidades para orientar y facilitar los procesos de crecimiento y desarrollo personal y vocacional de sus hijos. Estas sesiones estuvieron dirigidas especialmente al aumento de la participación familiar y comunitaria. En total, los mentores PCU implementaron 352 sesiones de mentoría con sus alumnos PCU ( $\mathrm{M}=17.6$; rango entre 13 y 23 sesiones por alumno PCU), 67 sesiones de con los docentes PCU $(M=3.35$; rango entre 1 y 8 sesiones por docente PCU de alumno PCU) y 84 sesiones con las familias ( $M$ $=5.25$; rango entre 1 y 13 sesiones por familia de alumno PCU).

Además, con la finalidad de vigilar posibles desviaciones del programa, se adoptaron diversas medidas de seguimiento global (registro y sesiones de seguimiento con agentes educativos implicados) y específico para las actividades del plan de intervención, es decir, para el seminario de formación (observadores participantes) y las sesiones de mentoría (tres medidas de seguimiento obligatorio): una sesión grupal entre responsables y los mentores PCU una vez desarrollada la segunda y quinta sesión y 178 sesiones individuales entre los responsables del programa y los mentores PCU $(M=9.4$; rango entre 2 y 20 sesiones por pareja de 
mentores PCU), solicitadas por los mentores PCU o los responsables del programa. En estas sesiones se valoró el desarrollo de las sesiones efectuadas a partir de los comentarios de los mentores PCU y el análisis de sus cuadernos, para seguidamente emitir las recomendaciones oportunas y analizar los objetivos y tareas a trabajar en próximas sesiones. Finalmente, para la evaluación de impacto del PCU se tomaron medidas pretest-postest de las variables dependientes, comprobando los efectos estadística y educativamente significativos.

Los datos para las diferentes hipótesis fueron analizados mediante las siguientes pruebas estadísticas: (a) Hipótesis 1 y 2 : z de Wilcoxon y $t$ para muestras relacionadas, tras efectuar la prueba de Kolmogorov-Smirnov, y se calculó el valor d de Cohen, (b) Hipótesis 3: estadístico de McNemar y (c) Hipótesis 4: estadísticos descriptivos y frecuencias.

\section{Resultados}

Los resultados de las comparaciones pretestpostest sobre estrategias de aprendizaje y motivación de los mentores PCU, Hipótesis 1, tras comprobar la ausencia de normalidad en la distribución de las puntuaciones, revelan diferencias estadísticamente significativas en la mayoría de las subescalas del MSLQ, oscilando el valor $d$ de Cohen entre - 0.1 puntos en Ansiedad y 0.89 puntos en Tiempo y ambiente de estudio (Tabla 1).
Tabla 1

Comparaciones intragrupo sobre estrategias de aprendizaje y motivación de mentores PCU

\begin{tabular}{|c|c|c|c|c|c|c|}
\hline Subescalas/Fases & $N$ & $M$ & $D E$ & $z$ & Significancia (2-colas) & $d$ \\
\hline \multicolumn{7}{|l|}{ Motivación } \\
\hline \multicolumn{7}{|l|}{ Orientación intrínseca } \\
\hline Pretest & 40 & $\begin{array}{l}5.79 \\
5.97\end{array}$ & 0.65 & -2.51 & \multirow[t]{2}{*}{$0.01^{*}$} & \multirow[t]{2}{*}{0.3} \\
\hline $\begin{array}{l}\text { Postest } \\
\text { Orientación extrinseca }\end{array}$ & 40 & 5.97 & 0.56 & & & \\
\hline Pretest & 40 & 4.88 & 1.37 & & \multirow[b]{2}{*}{0.12} & \multirow{2}{*}{-0.16} \\
\hline Postest & 40 & 4.65 & 1.54 & -1.55 & & \\
\hline \multicolumn{7}{|l|}{ Valor de la tarea } \\
\hline Pretest & 40 & 5.75 & 0.65 & & \multirow[t]{2}{*}{$0.04 *$} & \multirow[t]{3}{*}{0.42} \\
\hline Postest & 40 & 5.98 & 0.43 & -2.1 & & \\
\hline \multicolumn{6}{|c|}{$\begin{array}{l}\text { Control de las creencias de } \\
\text { aprendizaje }\end{array}$} & \\
\hline Pretest & 40 & 5.27 & 0.91 & -3.68 & \multirow{2}{*}{$0^{* * * *}$} & \multirow[t]{2}{*}{0.55} \\
\hline Postest & 40 & 5.69 & 0.59 & & & \\
\hline \multicolumn{7}{|l|}{ Autoeficacia } \\
\hline $\begin{array}{l}\text { Pretest } \\
\text { Potest }\end{array}$ & 40 & 5.92 & 0.6 & -3.64 & $0^{* * * *}$ & 0.47 \\
\hline \\
\hline Pretest & 40 & 2.87 & 1.37 & & \multirow{2}{*}{0.49} & \multirow{2}{*}{-0.1} \\
\hline Postest & 40 & 2.73 & 1.35 & -3.68 & & \\
\hline \multirow{2}{*}{\multicolumn{7}{|c|}{$\begin{array}{l}\text { Estrategias de aprendizaje } \\
\text { Pensamiento crítico }\end{array}$}} \\
\hline & & & & & & \\
\hline Pretest & 40 & 4.31 & 1 & -3.83 & \multirow{2}{*}{$0^{* * * *}$} & \multirow[t]{2}{*}{0.57} \\
\hline $\begin{array}{c}\text { Postest } \\
\text { Autorregulación metaco }\end{array}$ & 40 & 4.86 & 0.93 & & & \\
\hline Pretest & 40 & 4.67 & 0.64 & & \multirow[b]{2}{*}{$0^{* * *}$} & \multirow{2}{*}{0.82} \\
\hline Postest & 40 & 5.19 & 0.62 & -4.85 & & \\
\hline Tiempo y ambiente de $\mathrm{e}$ & & & & & & \\
\hline Pretest & 40 & 4.48 & 0.5 & & $0^{* * * *}$ & 0.89 \\
\hline $\begin{array}{l}\text { Postest } \\
\text { Estrategias de repaso }\end{array}$ & 40 & 4.9 & 0.44 & -3.45 & & \\
\hline $\begin{array}{l}\text { Estrategias de repaso } \\
\text { Pretest }\end{array}$ & & & & & & \\
\hline $\begin{array}{l}\text { Pretest } \\
\text { Postest }\end{array}$ & $\begin{array}{l}40 \\
40\end{array}$ & $\begin{array}{l}4.77 \\
5.37\end{array}$ & $\begin{array}{l}1.06 \\
0.85\end{array}$ & -4.7 & $0^{* * *}$ & 0.62 \\
\hline Estrategias de elaboracic & & & & & & \\
\hline Pretest & 40 & 5.64 & 0.6 & & $0^{* * * *}$ & 0.78 \\
\hline Postest & 40 & 6.05 & 0.43 & -4.1 & & \\
\hline Estrategias de organizac & & & & & & \\
\hline Pretest & 40 & 5.37 & 0.89 & -3.13 & $0^{* *}$ & 0.52 \\
\hline $\begin{array}{c}\text { Postest } \\
\text { Regulación del esfuerzo }\end{array}$ & 40 & 5.77 & 0.62 & & & \\
\hline Pretest & 40 & 3.61 & 0.55 & & & \\
\hline Postest & 40 & 3.73 & 0.6 & -1.06 & 0.29 & 0.21 \\
\hline Aprendizaje entre iguale & & & & & & \\
\hline Pretest & 40 & 4.57 & 1.3 & -1.45 & 0.15 & 0.14 \\
\hline $\begin{array}{l}\text { Postest } \\
\text { Púcolat }\end{array}$ & 40 & 4.76 & 1.35 & $-1,43$ & & \\
\hline Búsqueda de ayuda & & & & & & \\
\hline $\begin{array}{l}\text { Pretest } \\
\text { Postest }\end{array}$ & $\begin{array}{l}40 \\
40\end{array}$ & $\begin{array}{c}4.61 \\
5.4\end{array}$ & $\begin{array}{l}0.58 \\
0.65\end{array}$ & -3.83 & $0^{* * *}$ & -0.86 \\
\hline
\end{tabular}

En lo que respecta a la Hipótesis 2, comprobada la distribución normal de las puntuaciones, la prueba $t$ arroja diferencias estadísticamente significativas entre las puntuaciones medias obtenidas en la fase postest y pretest en gran parte de las escalas de la EHS (Gismero, 2000), con valores d que oscilan entre los 0.13 puntos en Defensa de los propios derechos y 0.68 puntos en Expresión de disconformidad (Tabla 2). 
Tabla 2

Comparaciones intragrupo sobre habilidades sociales de mentores PCU

\begin{tabular}{|c|c|c|c|c|c|c|}
\hline Escalas/Fases & $N$ & $M$ & $D E$ & $t$ & Significancia & $d$ \\
\hline \multicolumn{7}{|c|}{$\begin{array}{l}\text { Autoexpresión en situaciones } \\
\text { sociales }\end{array}$} \\
\hline Pretest & 40 & 24.97 & 4.61 & \multirow[b]{2}{*}{-6.59} & \multirow[b]{2}{*}{$0 * * *$} & \multirow{2}{*}{0.44} \\
\hline Postest & 40 & 26.87 & 3.98 & & & \\
\hline \multicolumn{7}{|c|}{ Expresión de disconformidad } \\
\hline Pretest & 40 & 13.02 & 1.64 & \multirow{2}{*}{-8.06} & \multirow{2}{*}{$0 * * *$} & \multirow{2}{*}{0.68} \\
\hline Postest & 40 & 14.15 & 1.69 & & & \\
\hline \multicolumn{7}{|c|}{ Decir no y cortar interacciones } \\
\hline Pretest & 40 & 18.6 & 3.26 & \multirow{2}{*}{-5.42} & \multirow{2}{*}{$0 * * *$} & \multirow{2}{*}{0.37} \\
\hline Postest & 40 & 19.8 & 3.26 & & & \\
\hline \multicolumn{7}{|l|}{ Hacer peticiones } \\
\hline Pretest & 40 & 15.65 & 3.38 & \multirow{2}{*}{-4.95} & \multirow{2}{*}{$0^{* * *}$} & \multirow[b]{2}{*}{0.40} \\
\hline Postest & 40 & 16.9 & 2.85 & & & \\
\hline \multicolumn{7}{|c|}{$\begin{array}{l}\text { Iniciar interacciones positivas } \\
\text { con el sexo opuesto }\end{array}$} \\
\hline Pretest & 40 & 13.72 & 4.01 & \multirow{2}{*}{-6.75} & \multirow{2}{*}{$0 * * *$} & \multirow{2}{*}{0.53} \\
\hline Postest & 40 & 15.77 & 3.72 & & & \\
\hline \multicolumn{7}{|c|}{ Defensa de los propios derechos } \\
\hline Pretest & 40 & 14.42 & 2.78 & \multirow{2}{*}{-1.41} & \multirow{2}{*}{0.15} & \multirow{2}{*}{0.13} \\
\hline Postest & 40 & 14.82 & 3.22 & & & \\
\hline \multicolumn{7}{|l|}{ PD global } \\
\hline Pretest & 40 & 100.4 & 14.59 & \multirow{2}{*}{-10.11} & \multirow{2}{*}{$0^{* * *}$} & \multirow{2}{*}{0.56} \\
\hline Postest & 40 & 108.32 & 13.81 & & & \\
\hline
\end{tabular}

En cuanto a la Hipótesis 3, el estadístico de McNemar no revela diferencias estadísticamente significativas entre el rendimiento escolar de los alumnos PCU en la evaluación del primer trimestre y el resto de evaluaciones del curso escolar (Tabla 3).

\section{Tabla 3}

Rendimiento escolar de alumnos PCU: número de áreas curriculares superadas y promoción de curso

\begin{tabular}{|c|c|c|c|c|c|c|c|c|c|}
\hline \multirow{2}{*}{ Casos } & \multicolumn{2}{|c|}{ Trimestre 1} & \multicolumn{2}{|c|}{ Trimestre 2} & \multicolumn{2}{|c|}{ Trimestre 3} & \multicolumn{2}{|c|}{ Evaluación Ordinaria } & \multirow[b]{2}{*}{ Promoción } \\
\hline & Apto & No Apto & Apto & No Apto & Apto & No Apto & Apto & No Apto & \\
\hline $1 \mathrm{EC}$ & 5 & 2 & 25 & 2 & 3 & 4 & 6 & & Promociona \\
\hline $2 \mathrm{EC}$ & 3 & 4 & 4 & 3 & 2 & 5 & 3 & & 4 Promociona \\
\hline $3 \mathrm{EC}$ & 6 & & 7 & 0 & 7 & 0 & 7 & & Promociona \\
\hline $4 \mathrm{EC}$ & 5 & 2 & 5 & 2 & 4 & 3 & 5 & & Promociona \\
\hline $5 \mathrm{EC}$ & 3 & 4 & 3 & 4 & 3 & 4 & 3 & & Repite \\
\hline $8 \mathrm{EC}$ & 3 & 3 & 4 & 2 & 4 & 2 & 3 & & Repite \\
\hline $9 \mathrm{EC}$ & 6 & 1 & 6 & 1 & 7 & 0 & 7 & & Promociona \\
\hline $10 \mathrm{EC}$ & 6 & & 7 & 0 & 7 & 0 & 7 & & Promociona \\
\hline $11 \mathrm{EC}$ & 5 & & 6 & 1 & 6 & 1 & 6 & & Promociona \\
\hline $12 \mathrm{EC}$ & 4 & & 6 & 1 & 6 & 1 & 7 & & Promociona \\
\hline $2 \mathrm{SC}$ & 4 & & 5 & 6 & 5 & 6 & 5 & & Repite \\
\hline $3 \mathrm{SC}$ & 4 & 3 & 4 & 3 & 5 & 2 & 5 & & Promociona \\
\hline 2LM & 5 & & 5 & 2 & 5 & 2 & 7 & & Promociona \\
\hline 3LM & 6 & 1 & 3 & 4 & 7 & 0 & 7 & & Promociona \\
\hline 4LM & 3 & & 3 & 4 & 3 & 4 & 3 & & Repite \\
\hline $1 \mathrm{GR}$ & 4 & 3 & 4 & 3 & 4 & 3 & 4 & & Repite \\
\hline $2 \mathrm{GR}$ & 4 & 3 & 5 & 2 & 5 & 2 & 6 & & Promociona \\
\hline 3GR & 5 & 2 & 5 & 2 & 5 & 2 & 6 & & Promociona \\
\hline $13 \mathrm{EC}$ & 7 & & 7 & 0 & 7 & 0 & 7 & 0 & Promociona \\
\hline $5 \mathrm{LM}$ & 5 & 2 & 5 & 2 & 5 & 2 & 5 & & 2 Promociona \\
\hline
\end{tabular}

Por último, respecto a la Hipótesis 4, los resultados revelan un notable nivel de satisfacción de los docentes PCU con el programa (Tabla 4).
Tabla 4

Satisfacción de docentes PCU

\begin{tabular}{|c|c|c|}
\hline Ítems & $N$ & $\%$ \\
\hline \multicolumn{3}{|c|}{$\begin{array}{l}\text { Ha notado cambios personales en su alumno/s } \\
\text { (apariencia, seguridad, higiene, relaciones, etc.) }\end{array}$} \\
\hline Sí & 13 & 65 \\
\hline No & 7 & 35 \\
\hline \multicolumn{3}{|c|}{$\begin{array}{l}\text { Ha notado cambios escolares en su alumno/s (atención, } \\
\text { autonomía, deberes, tareas, etc.) }\end{array}$} \\
\hline Sí & 17 & 85 \\
\hline No & 3 & 15 \\
\hline \multicolumn{3}{|c|}{ Los cambios han repercutido en sus calificaciones finales } \\
\hline Sí & 16 & 80 \\
\hline No & 4 & 20 \\
\hline \multicolumn{3}{|c|}{$\begin{array}{l}\text { Ha notado cambios en el comportamiento de los padres } \\
\text { (asistencia tutoría, interés, contacto, etc.) }\end{array}$} \\
\hline Sí & 15 & 75 \\
\hline No & 5 & 25 \\
\hline \multicolumn{3}{|c|}{ Si se replicase el programa, volvería a participar } \\
\hline Sí & 18 & 90 \\
\hline No & 2 & 10 \\
\hline Valoración global del PCU & $M=8.45$ & $D E=1.22$ \\
\hline
\end{tabular}

\section{Discusión}

El PCU fue diseñado y planificado como un conjunto de acciones coordinadas y dirigidas a mejorar la calidad de la oferta formativa en educación superior mediante su vinculación a la prevención del fracaso y/o abandono escolar temprano en etapas educativas obligatorias. En este sentido, tomando como referencia las hipótesis establecidas y los resultados obtenidos, se pueden establecer las siguientes conclusiones: (1) puesto que se aprecian diferencias estadísticamente significativas, y un elevado tamaño del efecto, entre las puntuaciones directas obtenidas por los mentores PCU en la fase postest en comparación con la fase pretest en estrategias de aprendizaje, motivación y habilidades sociales, las Hipótesis 1 y 2 se pueden aceptar parcialmente; (2) al no apreciarse diferencias estadísticamente significativas entre el rendimiento escolar logrado por los alumnos PCU en la evaluación del primer trimestre y el resto de evaluaciones del curso escolar, la Hipótesis 3 se rechaza y (3) debido a que el $90 \%$ de los docentes PCU manifestó estar dispuesto a participar en próximas ediciones del programa, la Hipótesis 4 no puede aceptarse.

Efectivamente, la participación en el programa ha tenido un efecto positivo y estadísticamente significativo sobre el grado de dominio de las estrategias de aprendizaje, nivel de motivación y habilidades sociales de los mentores 
Francisco D. Fernández-Martín, José L. Arco-Tirado, Mirian Hervás-Torres, et al.

PCU, y aunque no ha generado en los alumnos PCU, como grupo, un rendimiento escolar cercano a los objetivos y expectativas inicialmente establecidos, un elevado porcentaje de docentes PCU manifestó su intención de participar en próximas ediciones del programa. Estos resultados son bastante positivos, pues los objetivos planteados inicialmente eran puramente exploratorios, en los términos que recomienda la literatura especializada cuando se emplean este tipo de diseños metodológicos (Ato, 2010). De hecho, son aún más relevantes si se tiene en cuenta que en el caso de los mentores PCU, las variables dependientes se usaron como criterio para seleccionar a esta submuestra, por lo que las puntuaciones directas que ostentaban en la fase pretest eran bastante elevadas (efecto techo) (Arco \& Fernández, 2011). En el caso de los alumnos PCU, un análisis más exhaustivo de su rendimiento escolar, caso a caso, arroja tendencias de mejora en el 60 $\%$ de esta submuestra, destacando entre el 40 $\%$ restante de participantes la presencia de: (a) seis alumnos PCU que fueron evaluados con criterios de evaluación de su nivel o curso de referencia, cuando realmente a lo largo del curso escolar estuvieron trabajando contenidos de un nivel inferior al que cursaban, sin ser objeto de adaptación curricular, por lo que era improbable esperar mejoras en su rendimiento escolar y (b) dos alumnos PCU con necesidades personales atendidas por servicios sociales. Y, finalmente, en el caso de los docentes PCU, las dificultades de comunicación y coordinación que se presentaron con algunos de ellos.

Los cambios experimentados por las diferentes submuestras en las variables dependientes parecen confirmar las previsiones iniciales sobre los beneficios de participar en este tipo de programas, en términos muy similares a los que se especifica en la literatura especializada (Celio, Durlak, \& Dymnicki, 2011; DuBois, Portillo, Rhodes, Silverthorn, \& Valentine, 2011; Eby et al., 2008; Holsapple, 2012; Yorio \& Ye, 2012). Las nuevas experiencias cuasi-profesionales que los mentores PCU han vivido, los problemas y dificultades a los que se han enfrentado y resuelto, la práctica de hábitos profesionales como la participación en los procesos de toma de decisiones, el registro de datos, el seguimiento y la evaluación, la mayor concienciación sobre la necesidad e importancia del compromiso profesional con el trabajo, entre otros aspectos, generó beneficios en su desarrollo académico, personal y social. Igualmente, un porcentaje considerable de docentes PCU, manifestaron cambios personales y escolares en los alumnos PCU relacionados con su participación en el programa, incluso en las familias, en los mismos términos que mejoras en los aprendizajes y áreas curriculares, confirmando que éstas repercutieron finalmente en la calificaciones obtenidas por los alumnos PCU.

Ahora bien, para la interpretación de los resultados obtenidos es necesario considerar: (a) el $\mathrm{N}$ reducido de participantes que confirmaban las submuestras, (b) la posible presencia de algún sesgo producido por los diseños metodológicos empleados, (c) las diferencias en el número de sesiones llevadas a cabo por los mentores PCU, (d) las discrepancias entre las tareas realizadas en algunas de las sesiones de mentoría y las del aula ordinaria, incluso entre los materiales, como consecuencia de las dificultades de comunicación y coordinación con algunos docentes PCU y (e) la no realización por parte de algunos alumnos PCU de las tareas asignadas, o en su caso, la no supervisión de la realización de dichas tareas por parte de las familias, situación que se presentó en varias ocasiones en el caso de cuatro alumnos PCU.

Por tanto, en próximas ediciones del programa, de cara a incrementar la potencia de los resultados, sería conveniente seleccionar diseños metodológicos con mayor grado de experimentalidad, y ampliar el número de variables dependientes por considerar para las diferentes submuestras (rendimiento académico, clima escolar y familiar, etc.), con el propósito de alcanzar una mayor comprensión de la enorme heterogeneidad de beneficios y potencialidades de este tipo de programas (Clayton et al., 2013). Asimismo, con el propósito de mejorar los resultados obtenidos, también sería necesario introducir ciertos cambios en el diseño, planificación e implementación del PCU, como 
por ejemplo: (a) llevar a cabo las sesiones de mentoría en tiempo escolar, solucionando así las dificultades de comunicación y coordinación con los docentes PCU; (b) revisar los criterios de acceso y permanencia en el PCU, o en su caso, el perfil y compromiso de las submuestras y familias y (c) incluir medidas que permitan a los mentores PCU efectuar un número similar de sesiones (implementar las sesiones desde el inicio del curso escolar, lo que implicaría cambios en la temporización).

\section{Referencias}

Able, H., Ghulamani, H., Mallous, R., \& Glazier, J. (2014). Service learning. Connecting future teachers to the lives of diverse children and their families. Journal of Early Childhood Teacher Education, 35, 6-21. http s://doi.org/10.1080/10901027.2013.874383

Arco, J. L., \& Fernández, F. D. (2011). Eficacia de un programa de tutoría entre iguales para la mejora de los hábitos de estudio del alumnado universitario. Revista Psicodidáctica, 16(1), 163-180. Recuperado de http://www.ehu.eus/ojs/index.php/psico didactica/article/view/1131

Arco, J. L., Fernández, F. D., \& Fernández, J. M. (2011). The impact of a peertutoring program on quality standards in Higher Education. Higher Education, 62 (6), 773-788. https://doi.org/10.1007/s10734-0 11-9419-x

Ato, M. (2010). Tipología de los diseños cuasiexperimentales. En M. T. Anguera, J. Arnau, M. Ato, R. Martínez, J. Pascual \& G. Vallejo (Eds.), Métodos de investigación en Psicología (pp. 245-269). Madrid: Síntesis.

Billig, S., Root, S., \& Jesse, D. (2005). The impact of participation in service learning on high school students civic engagement. Denver, CO: RMC Research Corporation.

Bringle, R. G., Hatcher, J. A., \& Jones, S. G. (Eds.). (2011). International service learning. Conceptual frameworks and research. Sterling, VI: Stylus Publishing.
Clayton, P. H., Bringle, R. G., \& Hatcher, J. A. (Eds.). (2013). Research on service learning. Conceptual frameworks and assessment. Sterling, VI: Stylus Publishing.

Celio, C. I., Durlak, J., \& Dymnicki, A. (2011). A Meta-analysis of the impact of servicelearning on students. Journal of Experiential Education, 34(2), 164-181. https://doi.org/1 0.1177/105382591103400205

Comisión Europea. (2011). Agenda de nuevas cualificaciones y empleos: una contribución europea hacia el pleno empleo. Luxemburgo: Oficina de Publicaciones de la Unión Europea.

Comisión Europea. (2012). Un nuevo concepto de educación: invertir en las competencias para lograr mejores resultados económicos. Luxemburgo: Oficina de Publicaciones de la Unión Europea.

Consejo de la Unión Europea. (2009). Conclusiones del Consejo de 12 de mayo de 2009 sobre un marco estratégico para la cooperación europea en el ámbito de la educación y la formación ( $E$ ET 2020»). Luxembourg: Oficina de Publicaciones de la Unión Europea.

Consejo Económico y Social de España. (2015). Memoria sobre la situación socioeconómica y laboral de España 2015. Madrid: Autor.

Consejo Escolar del Estado. (2015). Informe 2015 sobre el estado del sistema educativo. Curso 2013-2014. Madrid: Ministerio de Educación, Cultura y Deporte.

DuBois, D. L., \& Karcher, M. J. (Eds.). (2014). Handbook of youth mentoring (2.a ed.). Thousand Oaks, CA: Sage.

DuBois, D. L., Portillo, N., Rhodes, J. E., Silverthorn, N., \& Valentine, J. C. (2011). How effective are mentoring programs for youth? A systematic assessment. Psychological Science in the Public Interest, 12(2), 57-91. https://doi.org/10.1177/1529 100611414806

Eby, L. T., Allen, T. D., Evans, S. C., Ng, T., \& DuBois, D. (2008). Does mentoring matter? A multidisciplinary meta-analysis comparing mentored and non-mentored individuals. Journal of Vocational Behavior, 
72(2), 254-267. https://doi.org/10.1016/j.jv b.2007.04.005

European University Association. (2015). EUA Public Funding Observatory 2015 (Informe). Recuperado de http://www.eua.be/Libraries /governance-autonomy-funding/november -2015.pdf?.sfrrsn $=0$

Gismero, E. (2000). Escala de Habilidades Sociales (EHS). Madrid: TEA.

Hayward, M. S. (2014). Service learning in the Community College: Drive to employment. Community College Journal of Research and Practice, 38(9), 838-841. https://doi.org/10. 1080/10668926.2013.790858

Holsapple, M. A. (2012). Service-learning and student diversity outcomes: Existing evidence and directions for future research. . Michigan Journal of Community Service Learning, 18(2), 5-18. Recuperado de http:/hdl.handle.net/2027/spo.3239521 .0018 .201

Instituto de la Empresa Familiar. (2011). Propuestas para el empleo juvenil en España (Informe). Recuperado de http://www.iefamiliar.com/upload/estati cos/o5e11v0nzw200bn1.pdf

Instituto Nacional de Evaluación Educativa. (2016). Sistema estatal de indicadores de la educación 2016 (Informe). Recuperado http://educalab.es/documents/ 10180/15845/2016SistemaEstataldeIndica doresdelaEducaci\%C3\%B3n.pdf/89e4c98e -dc7e-41d7-8084-25329329bab6

Martínez, R. (2010). El método de encuestas por muestreo: conceptos básicos. En M. T. Anguera, J. Arnau, M. Ato, R. Martínez, J. Pascual, \& G. Vallejo (Eds.), Métodos de investigación en Psicología (pp. 385-443). Madrid: Síntesis.

The National Mentoring Partnership (2014). Elements of effective practice for mentoring, 4th edition. Recuperado de https://www.mentoring.org/program-res ources/elements-of-effective-practice-formentoring/

Organisation for Economic Cooperation and Development. (2016). Education at a glance
2016: OECD Indicators. París: Autor. https ://doi.org/10.1787/eag-2016-en

Pintrich, P. R., Smith, D., García, T., \& McKeachie, W. (1991). Motivated Strategies for Learning Questionnaire (MSLQ). Michigan, MI: University of Michigan.

Tapia, M. N. (2006). Aprendizaje y servicio solidario en el sistema educativo y las organizaciones juveniles. Buenos Aires: Ciudad Nueva.

Van der Ende, R., Walsh, K., \& Ziminiene, N. (2014). European vacancy and recruitment report 2014. Luxembourg: Oficina de Publicaciones de la Unión Europea. https:/ /doi.org/10.2767/2563

Westover, J. H. (2012). Academic servicelearning across disciplines. Models, outcomes and assessment. Champaign, IL: Common Ground Publishing.

Yorio, P. L., \& Ye, F. (2012). A Meta-analysis of the effects of service-learning on the social, personal, and cognitive outcomes of learning. Academy of Management Learning $\mathcal{G}$ Educations, 11 (1), 9-27. https://doi.org/1 0.5465/amle.2010.0072

\section{Notas}

* Artículo de investigación. Este trabajo forma parte de un proyecto financiado parcialmente por el Ayuntamiento de Maracena (Granada). 